\title{
Quantum electrodynamics and photon-assisted tunnelling in long Josephson junctions
}

\author{
A.O. Sboychakov, ${ }^{1,2}$ Sergey Savel'ev, ${ }^{1,3}$ and Franco Nori ${ }^{1,4}$ \\ ${ }^{1}$ Frontier Research System, The Institute of Physical and Chemical Research (RIKEN), Wako-shi, Saitama, 351-0198, Japan \\ ${ }^{2}$ Institute for Theoretical and Applied Electrodynamics Russian \\ Academy of Sciences, Izhorskaya str. 13, Moscow 125412, Russia \\ ${ }^{3}$ Department of Physics, Loughborough University, Loughborough LE11 3TU, United Kingdom \\ ${ }^{4} M C T P, C S C S$, Department of Physics, University of Michigan, Ann Arbor, MI 48109, USA
}

(Dated: November 12, 2018)

\begin{abstract}
We describe the interaction between an electromagnetic field and a long Josephson junction (JJ) driven by a dc current. We calculate the amplitudes of emission and absorption of light via the creation and annihilation of quantized Josephson plasma waves (JPWs). Both, the energies of JPW quanta and the amplitudes of light absorption and emission, strongly depend on the junction's length and can be tuned by an applied dc current. Moreover, photon-assisted macroscopic quantum tunnelling in long Josephson junctions show resonances when the frequency of the outside radiation coincides with the current-driven eigenfrequencies of the quantized JPWs.
\end{abstract}

PACS numbers: $74.50 .+\mathrm{r}$

\section{INTRODUCTION}

The miniaturization of electronic devices allows the observation of quantum effects which were impossible to measure in the past. Systems of Josephson junctions, characterized by high frequency (up to several THz), exhibit a crossover to the quantum regime at relatively high temperatures. Indeed, quantum oscillations and macroscopic quantum tunnelling 1 (MQT) have been observed in charge, flux, and phase qubits ${ }^{2}$. Renewed interest in MQT occurred after the recent discovery of MQT in high temperature layered superconductors $3.4,5,6$. The observed enhancement of MQT was attributed to the spatial structure of the tunnelling fluxon 7.8 . It is important to develop a theory of quantum electrodynamics in long (about $1 \mu \mathrm{m}$ in stacks of JJs, and about tens of microns in low- $T_{c}$ junctions) JJJs where the spatial distribution of the gauge-invariant phase difference is crucial. In this problem, the standard quantum mechanical approach (where the phase difference is associated with the coordinate of a quantum particle tunnelling through an effective potential barrier) becomes invalid, and a more advanced field-theoretical approach is needed ${ }^{9,10}$.

Here we consider a Josephson junction (JJ) driven by a dc current near its critical value and exposed to $\mathrm{THz}$ electromagnetic (EM) waves. In this configuration, as known for point-like contacts, the probability of MQT depends on the intensity and frequency $\omega$ of the incident EM waves. In contrast to the short-junction case, we predict several resonant enhancements of the MQT escape rate, when the frequency $\omega$ matches the eigenfrequencies of the JPWs. We also propose a full quantum electrodynamical description of long JJs, to calculate the probabilities of absorption and emission of light by JPW quanta.

In section II we derive the model and quantize the field of the gauge-invariant phase difference $\varphi$. In section III we consider the interaction of the quantized $\varphi$ with photons and calculate the transition rates of absorbtion and emission of light by JPW quanta. This allows us to find the mean values of occupation numbers of JPW quanta and the mean energy of the system, which is pumped by external $\mathrm{THz}$ radiation. In section IV we calculate the probability of photon-assisted macroscopic quantum tunnelling of the phase difference.

\section{SECOND QUANTIZATION OF THE PHASE DIFFERENCE FIELD}

\section{A. Lagrangian formulation}

The geometry of the Josephson junction (JJ) under study is shown in Fig. 1. Two superconducting bars overlap a length $D$ in the $x$ direction. An insulating layer of thickness $s$, about several nanometers, is placed between these two bars. A supercurrent with density $i$ flows through the junction in the $z$ direction. The width $L$ of the JJ in the $y$ direction is of the order of, or less than, the Josephson penetration depth $\lambda_{J}$, that is $l=L / \lambda_{J} \lesssim 1$. The dynamics of the gauge-invariant phase difference $\varphi(t, x, y)$ of such a junction is described by the action

$$
\begin{aligned}
\mathcal{S}[\varphi] & =\frac{1}{\omega_{p}} \int d t\left(\mathcal{L}[\varphi]+\mathcal{L}_{\Sigma}[\varphi]\right), \\
\mathcal{L}[\varphi] & =\frac{\lambda_{J} E_{J}}{L} \int d x d y\left[\frac{1}{2}\left(\frac{\partial \varphi}{\partial t}\right)^{2}-\frac{1}{2}(\boldsymbol{\nabla} \varphi)^{2}+\cos \varphi\right], \\
\mathcal{L}_{\Sigma}[\varphi] & =\frac{c E_{J}}{4 \pi i_{c} L} \oint_{\Sigma} d \zeta \varphi\left[\mathbf{H} \times \mathbf{e}_{z}\right]_{n} .
\end{aligned}
$$

In these equations, the $x$ and $y$ coordinates are normalized by $\lambda_{J}$, the time $t$ is normalized by $1 / \omega_{p}$, where $\omega_{p}$ is the Josephson plasma frequency, $i_{c}$ is the critical current density, and

$$
E_{J}=\hbar \omega_{p} \Lambda, \Lambda=\frac{i_{c} \lambda_{J} L}{2 e \omega_{p}}
$$


where $\Lambda$ is considered to be much larger than unity, $\Lambda \gg$ 1. The integration in $\mathcal{L}_{\Sigma}[\varphi]$ is performed over a contour $\Sigma$ around the junction's area, and the subscript $n$ refers to the component normal to the contour $\Sigma$ in the $X Y$ plane of the vector product of the magnetic field $\mathbf{H}$ and unit vector $\mathbf{e}_{z}$.

The classical equation of motion for $\varphi$ with the action (1) is the two dimensional Sine-Gordon equation

$$
\frac{\partial^{2} \varphi}{\partial t^{2}}-\triangle \varphi+\sin \varphi=0
$$

The surface term in the action (1D) depicts the boundary conditions to this equation,

$$
\left.\frac{\partial \varphi}{\partial n}\right|_{\mathbf{r} \in \Sigma}=\left[\mathbf{H} \times \mathbf{e}_{z}\right]_{n} .
$$

Representing the magnetic field in the form $\mathbf{H}=\mathbf{H}_{J}+$ $\mathbf{H}^{e}$, where $\mathbf{H}^{e}$ is the external ac magnetic field and $\mathbf{H}_{J}$ is the field generated by the flowing current, we obtain

$$
\begin{aligned}
& \left.\frac{\partial \varphi}{\partial x}\right|_{x= \pm d / 2}= \pm \frac{I}{2}+\frac{c H_{y}^{e}}{4 \pi i_{c} \lambda_{J}}, \\
& \left.\frac{\partial \varphi}{\partial y}\right|_{y= \pm l / 2}=-\frac{c H_{x}^{e}}{4 \pi i_{c} \lambda_{J}}
\end{aligned}
$$

where

$$
I=\frac{i D}{i_{c} \lambda_{J}}, \quad d=\frac{D}{\lambda_{J}}, \quad l=\frac{L}{\lambda_{J}} .
$$

When $\mathbf{H}^{e}=0$, the stationary solution to Eq. (2), corresponding to the lowest-energy minimum, does not depend on the $y$-coordinate. Below we assume that the ac magnetic field of the incident radiation is directed along the $y$-axis (see Fig. 1). In this case, only the plasma waves in the $x$-direction are excited, and $\varphi=\varphi(t, x)$. When $D \ll \lambda_{J}$, the field $\varphi(t, x)$ only slightly depends on the $x$-coordinate, and the action (11) describes the dynamics of the particle in the washboard potential $V(\varphi)=-\cos \varphi-j \varphi$, where $j=i / i_{c}$. When $j<1$, this potential has an infinite number of minima, each one separated by a potential barrier of the order of $\sqrt{1-j^{2}}$. The probability of quantum tunnelling from one minimum to the nearest minimum can be easily calculated in the semiclassical approximation ${ }^{11}$. When $D \gtrsim \lambda_{J}$, the spatial dependence of the field $\varphi(t, x)$ is essential, and the problem of quantum tunnelling becomes more complicated. In the semiclassical approximation, the probability of tunnelling can be written as 12

$$
\Gamma=\omega_{0} \sqrt{\frac{30 B}{\pi}} \exp (-B),
$$

where $B=2 \mathcal{S}_{\mathrm{E}} / \hbar$, and $\mathcal{S}_{\mathrm{E}}$ is the action, defined in Eq. (11), in imaginary time $t=i \tau$ calculated along classical trajectories, and $\omega_{0}$ is the oscillation frequency of the field $\varphi(t, x)$ near one of the energy minima $\varphi_{0}(x)$.

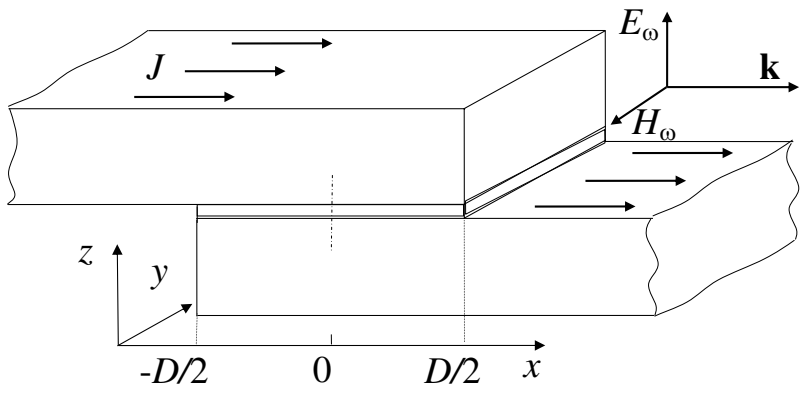

FIG. 1: Schematic diagram of the Josephson junction. The wave vector of the externally applied polarized $\mathrm{THz}$ electromagnetic wave is directed along the $x$ axis, while its electric (magnetic) field is directed along the $z(y)$ axis.

In one of our previous papers ${ }^{9}$ we proposed an approach for calculating the tunnelling exponent $B$ for a current $I=j d$ close to the critical value $I_{c}(d)$ (which now nonlinearly depends on $d$ ). Here we consider the effect of external electromagnetic radiation on the probability of tunnelling.

\section{B. Quantum regime}

We consider the interaction of $\varphi$ with electromagnetic waves by perturbations. First, we quantize the field $\varphi(t, x)$ near the energy minimum $\varphi_{0}(x)$ at $\mathbf{H}^{e}=0$, find the energy spectrum, and then calculate the transition rates of the field $\varphi$, from the ground state to its excited states and vice versa, due to the interaction with the electromagnetic field. The knowledge of the transition rates gives us the mean energy $\bar{E}(\omega, P)$ of the field $\varphi$ in the presence of an external radiation as a function of its power $P$ and frequency $\omega$. Since the effective potential barrier decreases with the growth of $\bar{E}$, the external radiation enhances the tunnelling. It is clear that a strong enhancement of the escape rate $\Gamma$ should be at frequencies $\omega$ close to the eigenfrequencies $\omega_{n}$ of the $\varphi$ field. The tunnelling exponent $B$, as function of $\bar{E}$, is found here using the approach described in Ref. 9 .

The static solution corresponding to an energy minimum satisfies the static Sine-Gordon equation

$$
\frac{d^{2} \varphi_{0}}{d x^{2}}=\sin \varphi_{0}
$$

with the boundary conditions

$$
\left.\frac{d \varphi_{0}}{d x}\right|_{x= \pm d / 2}= \pm \frac{I}{2} .
$$

The solution to this equation exists for currents $I$ less than the critical value $I_{c}(d)$. If $d \lesssim 4 \lambda_{J}$, the current density in the JJ is approximately constant and the function $I_{c}(d)$ increases linearly with $d$; if $d \gg \lambda_{J}$, the current 
flows near the junction edges and $I_{c}(d)$ reaches the saturation value $I_{c}^{\max }=4$. In order to quantize $\varphi$ we represent it in the form

$$
\hat{\varphi}(t, x)=\varphi_{0}(x)+\hat{\psi}(t, x),
$$

where the operator $\hat{\psi}$ satisfies the boundary conditions

$$
\left.\frac{d \hat{\psi}}{d x}\right|_{x= \pm d / 2}=0
$$

and expand the Lagrangian $\mathcal{L}$ in powers of $\hat{\psi}$. We introduce the momentum

$$
\hat{\pi}(t, x)=\frac{\delta \mathcal{L}}{\delta\left(\frac{\partial \hat{\psi}(t, x)}{\partial t}\right)}=\hbar \omega_{p} \Lambda \frac{\partial \hat{\psi}(t, x)}{\partial t}
$$

and require the standard simultaneous commutation relation

$$
\left[\hat{\psi}(t, x), \hat{\pi}\left(t, x^{\prime}\right)\right]_{-}=i \hbar \delta\left(x-x^{\prime}\right)
$$

The Hamiltonian of the system, $\hat{\mathcal{H}}$, has a form

$$
\hat{\mathcal{H}}=\hat{\pi} \frac{\partial \hat{\psi}}{\partial t}-\hat{\mathcal{L}}=\hat{\mathcal{H}}_{0}+\hat{\mathcal{H}}^{\prime}
$$

where

$$
\begin{aligned}
& \hat{\mathcal{H}}_{0}=E_{J}: \int_{-d / 2}^{d / 2} d x\left[\frac{1}{2 E_{J}^{2}} \hat{\pi}^{2}+\frac{1}{2} \hat{\psi} \hat{\mathcal{D}} \hat{\psi}\right]: \\
& \hat{\mathcal{H}}^{\prime}=E_{J}: \int_{-d / 2}^{d / 2} d x\left[\frac{1}{6} \sin \varphi_{0} \hat{\psi}^{3}+\frac{1}{24} \cos \varphi_{0} \hat{\psi}^{4}+\ldots\right]: .
\end{aligned}
$$

In these equations, the colons ":" mean "normal ordering" and $\hat{\mathcal{D}}$ is a differential operator of the form

$$
\hat{\mathcal{D}}=-\frac{\partial^{2}}{\partial x^{2}}+\cos \left(\varphi_{0}(x)\right) .
$$

In the interaction representation, the operators $\hat{\psi}$ and $\hat{\pi}$ can be written as

$$
\begin{aligned}
& \hat{\psi}=\sqrt{\frac{1}{2 \Lambda}} \sum_{n} \frac{\psi_{n}(x)}{\mu_{n}^{1 / 4}}\left(\mathrm{e}^{i \sqrt{\mu_{n}} t} \hat{b}_{n}^{\dagger}+\mathrm{e}^{-i \sqrt{\mu_{n}} t} \hat{b}_{n}\right), \\
& \hat{\pi}=i \hbar \omega_{p} \sqrt{\frac{\Lambda}{2}} \sum_{n} \frac{\psi_{n}(x)}{\mu_{n}^{1 / 4}}\left(\mathrm{e}^{i \sqrt{\mu_{n}} t} \hat{b}_{n}^{\dagger}-\mathrm{e}^{-i \sqrt{\mu_{n}} t} \hat{b}_{n}\right),
\end{aligned}
$$

where $\mu_{n}, \psi_{n}$ are, respectively, the eigenvalues and orthogonal eigenfunctions of the operator $\hat{\mathcal{D}}$, that is,

$$
\hat{\mathcal{D}} \psi_{n}=\mu_{n} \psi_{n}, \quad \int_{-d / 2}^{d / 2} d x \psi_{n}(x) \psi_{m}(x)=\delta_{n m}
$$

In Eq. (13), $\hat{b}_{n}^{\dagger}$ and $\hat{b}_{n}$ are the creation and annihilation operators of JPW quanta in the state $n$. Note that all $\mu_{n}$ 's are positive when $I<I_{c}(d)$ because the $\varphi_{0}(x)$ corresponds to an energy minimum. In terms of the operators $\hat{b}_{n}^{\dagger}$ and $\hat{b}_{n}$, the Hamiltonian $\hat{\mathcal{H}}_{0}$ takes the form

$$
\hat{\mathcal{H}}_{0}=\hbar \omega_{p} \sum_{n} \sqrt{\mu_{n}} \hat{b}_{n}^{\dagger} \hat{b}_{n} .
$$

The Hamiltonian $\hat{\mathcal{H}}^{\prime}$ describes the self-interaction of the field $\varphi$. Since $\Lambda \gg 1, \hat{\mathcal{H}}^{\prime}$ can be considered as a perturbation if the energy of the system (counting from the 'vacuum' state corresponding to $\left.\varphi_{0}(x)\right) \bar{E} \ll \hbar \omega_{p} \Lambda$. In zeroth order, this energy is determined by the occupation numbers $N_{n}$, and reads

$$
\bar{E}=\hbar \omega_{p} \sum_{n} \sqrt{\mu_{n}} N_{n}
$$

The correction to this result due to self interactions can be found via perturbation theory.

\section{INTERACTION WITH AN ELECTROMAGNETIC FIELD: ABSORPTION AND EMISSION TRANSITION RATES}

Now we consider the interaction of the field $\varphi$ with electromagnetic waves, described by the vector potential $\mathbf{A}$ (we choose the gauge $A_{0}=0, \operatorname{div} \mathbf{A}=0$ ). Substituting $\varphi=\varphi_{0}+\psi$ into the action (1) and expanding it in a power series of $\psi$, we derive the operator $\hat{V}$ describing the interaction of $\psi$ with the electromagnetic field ${ }^{16}$ :

$$
\hat{V}=-\frac{c E_{J}}{4 \pi i_{c} \lambda_{J} L} \oint_{\Sigma} d \zeta \hat{\psi}\left[\operatorname{rot} \mathbf{A} \times \mathbf{e}_{z}\right]_{n} .
$$

Here we use the relation $\mathbf{H}^{e}=\operatorname{rot} \mathbf{A} / \lambda_{J}$ because we measure distances in units of $\lambda_{J}$. The vector potential $\mathbf{A}$ in Eq. (16) consists of two parts, describing both the incoming and outgoing radiation re-emitted by the JJ. We assume that the incident electromagnetic radiation is fully polarized and propagates along the $x$ axis, as shown in Fig. 1. Below we measure the frequency $\omega$ in units of $\omega_{p}$ and the wave length in units of $\lambda_{J}$. In this case, $\mathbf{A}$ can be written as

$$
\begin{aligned}
\hat{\mathbf{A}}(t, \mathbf{r})= & -i \mathbf{e}_{z} \frac{c}{\omega_{p}} \int \frac{d \omega}{2 \pi} \frac{E_{\omega}}{\omega} \mathrm{e}^{-i \omega(t-v x)}+ \\
& \sqrt{\frac{4 \pi c^{2}}{V \omega_{p}}} \sum_{\mathbf{k}, \lambda}\left(\sqrt{\frac{\hbar}{2 \omega_{\mathbf{k}}}} \mathrm{e}^{\lambda}(\mathbf{k}) \mathrm{e}^{-i \omega_{\mathbf{k}} t+i \mathbf{k r}} \hat{a}_{\mathbf{k} \lambda}+\right. \\
& \left.\sqrt{\frac{\hbar}{2 \omega_{\mathbf{k}}}} \mathrm{e}^{\lambda}(\mathbf{k}) \mathrm{e}^{i \omega_{\mathbf{k}} t-i \mathbf{k} \mathbf{r}} \hat{a}_{\mathbf{k} \lambda}^{\dagger}\right)
\end{aligned}
$$

where $\hat{a}_{\mathbf{k} \lambda}^{\dagger}$ and $\hat{a}_{\mathbf{k} \lambda}$ are the creation and annihilation operators of a photon with wave vector $\mathbf{k}$ and polarization $\lambda$

$$
v=\frac{\omega_{p} \lambda_{J}}{c}
$$


is the ratio of the Swihart velocity $\omega_{p} \lambda_{J}$ to the speed of light $c, \omega_{\mathbf{k}}=|\mathbf{k}| / v$, and $V$ is the volume of space (dimensional) where the electromagnetic field exists. The first term in Eq. (17) corresponds to incoming radiation (which is here considered as classical), where $E_{\omega}$ is the electric field at frequency $\omega$. The second term describes the photons appearing due to the interaction of the incoming electromagnetic waves with the JJ. In this term, $\mathbf{e}^{\lambda}$ is the vector of polarization, which satisfies the equality

$$
\mathbf{k} \cdot \mathbf{e}^{\lambda}(\mathbf{k})=0
$$

Substituting Eq. (17) and the expansion (13) for $\hat{\psi}$ into Eq. (16), and performing the surface integration, we derive:

$$
\begin{aligned}
& \hat{V}=\hat{V}_{\text {ext }}+\hat{V}_{\mathrm{q}} \\
& \hat{V}_{\mathrm{ext}}= i \sqrt{\frac{\hbar c \lambda_{L} L v}{4 \pi}} \sum_{n} \int \frac{d \omega}{2 \pi} E_{\omega} \frac{\varkappa_{n}(\omega v)}{\sqrt{\omega_{n}}} \mathrm{e}^{-i \omega t} \times \\
&\left(\mathrm{e}^{i \omega_{n} t} \hat{b}_{n}^{\dagger}+\mathrm{e}^{-i \omega_{n} t} \hat{b}_{n}\right) \\
& \hat{V}_{\mathrm{q}}= \hbar c \sqrt{\frac{\lambda_{L} L}{2 V \lambda_{J}}} \sum_{n} \sum_{\mathbf{k} \lambda} \frac{S\left(k_{y}\right)}{\sqrt{\omega_{\mathbf{k}} \omega_{n}}} \times \\
&\left(\left[k_{y} h_{x}^{\lambda}(\mathbf{k}) \chi_{n}\left(k_{x}\right)+h_{y}^{\lambda}(\mathbf{k}) \varkappa_{n}\left(k_{x}\right)\right] \mathrm{e}^{-i \omega_{\mathbf{k}} t} \hat{a}_{\mathbf{k} \lambda}+\right. \\
& {\left.\left[k_{y} h_{x}^{\lambda}(\mathbf{k}) \chi_{n}^{*}\left(k_{x}\right)+h_{y}^{\lambda}(\mathbf{k}) \varkappa_{n}^{*}\left(k_{x}\right)\right] \mathrm{e}^{i \omega_{\mathbf{k}} t} \hat{a}_{\mathbf{k} \lambda}^{\dagger}\right) \times } \\
&\left(\mathrm{e}^{i \omega_{n} t} \hat{b}_{n}^{\dagger}+\mathrm{e}^{-i \omega_{n} t} \hat{b}_{n}\right)
\end{aligned}
$$

where $\lambda_{L}$ is the London penetration depth, which is linked to $i_{c}$ and $\lambda_{J}$ by the relation (it is supposed here that $\left.s \ll \lambda_{L}\right)$

$$
\lambda_{J}^{2}=\frac{\hbar c^{2}}{8 \pi e\left(2 \lambda_{L}+s\right) i_{c}} \cong \frac{\hbar c^{2}}{16 \pi e \lambda_{L} i_{c}} .
$$

In formulas (19) and (20)

$$
\begin{gathered}
\omega_{n}=\sqrt{\mu_{n}}, \\
\mathbf{h}^{\lambda}(\mathbf{k})=\mathbf{k} \times \mathbf{e}^{\lambda}(\mathbf{k}),
\end{gathered}
$$

and functions $\varkappa_{n}(k), \chi_{n}(k)$, and $S(k)$ are the following:

$$
\begin{gathered}
\varkappa_{n}(k)=-i\left[\psi_{n}(d / 2) \mathrm{e}^{i k d / 2}-\psi_{n}(-d / 2) \mathrm{e}^{-i k d / 2}\right], \\
\chi_{n}(k)=\int_{-d / 2}^{d / 2} d x \psi_{n}(x) \mathrm{e}^{i k x} \\
S(k)=\frac{2 \sin (k l / 2)}{k l} .
\end{gathered}
$$

\section{A. Spontaneous photon emission}

In first order of perturbation theory, there are only three possible processes: (i) spontaneous emission of a photon by the field $\varphi$, (ii) induced photon absorption, and (iii) induced emission ${ }^{17}$. Let us first consider the spontaneous emission, which is described by the operator $\hat{V}_{q}$. In the initial state, we have the set of occupation numbers of JPW quanta, $\left\{N_{m}\right\}$, and zero photons, and, in the final state, one of these numbers, say $N_{n}$, decreases by 1 and one photon appears in the system. We neglect the effect of thermal radiation, proceeding to the limit $T \rightarrow 0$. The probability per unit time of such a process, $w^{(-)}$, is proportional to $N_{n}$.

Following the standard approach ${ }^{13}$, we derive for the probability of emission of a photon with wave vector $\mathbf{k}$ and polarization $\lambda$ :

$$
d w_{n \lambda}^{(-)}(\mathbf{k})=N_{n} \frac{\pi c \lambda_{L} L}{v \lambda_{J}^{3}} \frac{\left|F_{n}^{\lambda}(\mathbf{k})\right|^{2}}{\omega_{\mathbf{k}} \omega_{n}} \delta\left(\omega_{\mathbf{k}}-\omega_{n}\right) \frac{d^{3} \mathbf{k}}{(2 \pi)^{3}},
$$

where

$$
F_{n}^{\lambda}(\mathbf{k})=S\left(k_{y}\right)\left[k_{y} h_{x}^{\lambda}(\mathbf{k}) \chi_{n}\left(k_{x}\right)+h_{y}^{\lambda}(\mathbf{k}) \varkappa_{n}\left(k_{x}\right)\right] .
$$

We introduce spherical coordinates in momentum space. Performing the integration over $\mathbf{k}$ and the summation over $\lambda$ taking into account the relation (18), finally, we derive

$$
\begin{gathered}
w_{n}^{(-)}=N_{n} \omega_{p} \gamma_{n}, \quad \gamma_{n}=\frac{\lambda_{L} L v}{2 \pi \lambda_{J}^{2}} \nu_{n}, \\
\nu_{n}=\frac{1}{4 \pi} \sum_{\lambda} \int d \mathbf{m}\left|F_{n}^{\lambda}\left(\omega_{n} v \mathbf{m}\right)\right|^{2},
\end{gathered}
$$

where $\mathbf{m}$ is a unit vector in momentum space.

For not too long junctions, it is possible to obtain an analytical expression for $\nu_{n}$. The wave length $\lambda$ of the electromagnetic radiation under consideration is about

$$
\lambda \sim c / \omega_{p}=\lambda_{J} / v \gg \lambda_{J},
$$

since the typical value of $v \sim 3 \cdot 10^{-2} \ll 1$. Therefore, for $d \ll \lambda$, one can expand $F_{n}^{\lambda}\left(\omega_{n} v \mathbf{m}\right)$ in Eq. (29) in powers of $v$. Doing so, we derive in the lowest order

$$
\begin{aligned}
\nu_{2 m+1} & \cong \frac{8}{3} \omega_{2 m+1}^{2} v^{2} \psi_{2 m+1}^{2}\left(\frac{d}{2}\right) \\
\nu_{2 m} & \cong \frac{4 d^{2}}{15} \omega_{2 m}^{4} v^{4}\left[\psi_{2 m}^{2}\left(\frac{d}{2}\right)-\frac{1}{2} \bar{\psi}_{2 m} \psi_{2 m}\left(\frac{d}{2}\right)+\bar{\psi}_{2 m}^{2}\right]
\end{aligned}
$$

where

$$
\bar{\psi}_{n}=\frac{1}{d} \int_{-d / 2}^{d / 2} d x \psi_{n}(x)
$$

and $\nu_{2 m} \ll \nu_{2 m+1}$. The difference between $\nu_{n}$ with odd and even $n$ comes from the symmetry properties of the JPW wave functions: $\psi_{n}(-x)=(-1)^{n} \psi_{n}(x)$. 
The value of $\gamma_{n}$ gives us the radiation width of the $n$th level in units of $\omega_{p}$. When $d \sim \lambda_{J}$, we have from (30) $\nu_{2 m+1} \sim v^{2}$ and $\nu_{2 m} \sim v^{4}$. Considering $L, \lambda_{J} \sim 10^{-3} \mathrm{~cm}$, $\lambda_{L} \sim 10^{-5} \mathrm{~cm}$, and $v \sim 3 \cdot 10^{-2}$ we obtain $\gamma_{2 m+1} \sim$ $10^{-8}-10^{-7}$ and $\gamma_{2 m} \sim 10^{-12}-10^{-10}$. Note that we do not consider here another possible mechanisms of dissipation, which can substantially increase the width of the JPW quanta energy levels.

\section{B. Induced photon absorbtion and emission}

Let us now consider processes of induced photon absorption and emission. These two processes are determined by the operator $\hat{V}_{\text {ext }}$. We denote by ${ }^{\text {in }} w_{n}^{(+)}$ (in $w_{n}^{(-)}$) the probability per unit time of creation (annihilation) of a quantum of the $\varphi$ field in the $n$th state due to induced photon absorption (emission). These two probabilities satisfy the following equality

$$
\frac{{ }^{\text {in }} w_{n}^{(+)}}{{ }^{\mathrm{in}} w_{n}^{(-)}}=\frac{N_{n}+1}{N_{n}}
$$

Thus, to first order in perturbation theory, the probability per unit time of induced photon absorption and also accounting for induced emission, $w_{n}^{(+)}={ }^{\text {in }} w_{n}^{(+)}{ }^{\text {in }} w_{n}^{(-)}$, does not depend on $N_{n}$, and is only determined by the power and frequency of the external radiation. Making a similar calculation as for $w_{n}^{(-)}$, we derive

$$
w_{n}^{(+)}=\frac{c \lambda_{L} L v}{2 \hbar \omega_{p}} \int d \omega \frac{\left|E_{\omega}\right|^{2}}{2 \pi} \frac{\left|\varkappa_{n}(\omega v)\right|^{2}}{\omega_{n}} \delta\left(\omega-\omega_{n}\right) .
$$

We assume that the incident radiation has a Gaussian distribution with central frequency $\bar{\omega}$ and width $\bar{\gamma}$, that is

$$
\frac{\left|E_{\omega}\right|^{2}}{2 \pi}=\frac{4 \pi P}{c} \Gamma(\omega-\bar{\omega})
$$

where $P$ is the radiation power per unit area and

$$
\Gamma(\omega)=\frac{1}{\bar{\gamma} \sqrt{\pi}} \exp \left\{-\omega^{2} / \bar{\gamma}^{2}\right\}
$$

The probability $w_{n}^{(+)}$then becomes

$$
w_{n}^{(+)}=\frac{2 \pi P \lambda_{L} L v}{\hbar \omega_{p}} \frac{f_{n}(\bar{\omega})}{\omega_{n}}
$$

where

$$
f_{n}(\bar{\omega})=\int d \omega \Gamma(\omega-\bar{\omega})\left|\varkappa_{n}(\omega v)\right|^{2} \delta\left(\omega-\omega_{n}\right) .
$$

In equilibrium, the probabilities $w_{n}^{(+)}$and $w_{n}^{(-)}$coincide. This gives rise to a relation for the mean values of the occupation numbers $\bar{N}_{n}$ :

$$
\bar{N}_{n}=\frac{4 \pi^{2} P \lambda_{J}^{2}}{\hbar \omega_{p}^{2}} \frac{f_{n}(\bar{\omega})}{\omega_{n} \nu_{n}}
$$

The mean value $\bar{E}$ of the system energy (to zeroth order in $\hat{\mathcal{H}}^{\prime}$ ) then reads

$$
\bar{E}=\frac{4 \pi^{2} P \lambda_{J}^{2}}{\omega_{p}} \sum_{n} \frac{f_{n}(\bar{\omega})}{\nu_{n}} .
$$

If the frequency band of the radiation source is large enough, that is, $\bar{\gamma} \gg \gamma_{n}$, we can easily perform an integration in Eq. (36). As a result, the mean energy becomes

$$
\bar{E}=\frac{4 \pi^{2} P \lambda_{J}^{2}}{\omega_{p}} \sum_{n} \frac{\left|\varkappa_{n}\left(\omega_{n} v\right)\right|^{2}}{\nu_{n}} \Gamma\left(\bar{\omega}-\omega_{n}\right) .
$$

In the opposite case of near-monochromatic radiation, $\bar{\gamma} \ll \gamma_{n}$, we should take into account that the energy levels of JPW quanta have finite width $\gamma_{n}$ (in units of $\left.\omega_{p}\right)$. Replacing the delta function in Eq. (36) by

$$
\delta\left(\omega-\omega_{n}\right) \rightarrow \frac{1}{\pi} \frac{\gamma_{n}}{\left(\omega-\omega_{n}\right)^{2}+\gamma_{n}^{2}}
$$

and using $\Gamma(\omega-\bar{\omega})=\delta(\omega-\bar{\omega})$, we obtain

$$
\bar{E}=\frac{2 P \lambda_{L} L v}{\omega_{p}} \sum_{n} \frac{\left|\varkappa_{n}\left(\omega_{n} v\right)\right|^{2}}{\left(\bar{\omega}-\omega_{n}\right)^{2}+\gamma_{n}^{2}},
$$

where we take into account that $\gamma_{n} \ll 1$. Let us notice that formulas (38), (39), and (40) are valid only at not too high radiation power when $\bar{E} \ll \hbar \omega_{p} \Lambda$.

\section{Response of the junction to a wave packet}

Consider now the response of a JJ to a wide-band $\mathrm{THz}$ wave packet. We now assume that the central frequency of the incoming radiation $\bar{\omega}$ is about $\omega_{p}$ and that the width $\bar{\gamma}$ of the wave packet is large enough. In this case, the first several energy levels of the system will be excited. The intensity $U(\omega)$ of light re-emission at frequency $\omega$ is given by the sum $\hbar \omega_{p} \sum_{n \lambda} \omega_{n} d w_{n \lambda}^{(-)}(\mathbf{k})$, with $d w_{n \lambda}^{(-)}(\mathbf{k})$ from Eq. (26), integrated over all directions of k. Taking into account the relation (37) for the mean values of the occupation numbers $\bar{N}_{n}$, and replacing again the delta function in Eq. (26) by a Lorentzian curve, we obtain

$$
U(\omega)=2 P \lambda_{L} L \sum_{n} \frac{\left|\varkappa\left(\omega_{n} v\right)\right|^{2} \Gamma\left(\bar{\omega}-\omega_{n}\right)}{\left(\omega-\omega_{n}\right)^{2}+\gamma_{n}^{2}}
$$

The function $U(\omega)$, for relatively short $(d=2)$ and long JJs $(d=5)$, is shown in Fig. 2 The wave packet of the incident radiation has a central frequency $\bar{\omega}=1$ and width $\bar{\gamma}=0.2$ (in units of $\omega_{p}$ ). In this case, the first two $(d=2)$ or three $(d=5)$ energy levels are excited. For short junctions, $d \lesssim 1$, the eigenfrequencies are, approximately

$$
\omega_{0} \approx\left(1-j^{2}\right)^{1 / 4}, \quad \omega_{n} \approx \frac{\pi n}{d} \gg \omega_{0}, n>0 .
$$




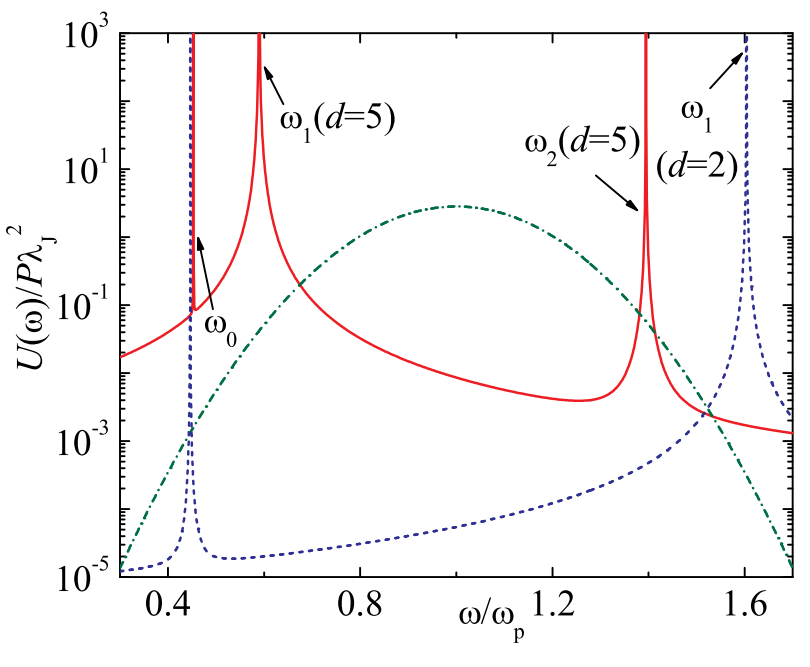

FIG. 2: (Color online) The frequency dependence of the intensity of re-emission, $U(\omega)$, calculated for $d=5$ (red solid curve) and $d=2$ (blue dashed curve). Other parameters are: $I / I_{c}(d)=0.98, \lambda_{L} / \lambda_{J}=4 \cdot 10^{-3}$, and $L / \lambda_{J}=2$. The green dot-dashed curve corresponds to Gaussian distribution of the intensity of incoming radiation with central frequency $\bar{\omega}=1$ and the width $\bar{\gamma}=0.2$ (in units of $\omega_{p}$ ).

For increasing values of $d, \omega_{1}$ tends to $\omega_{0}$, and when $d \gtrsim 4$, we have

$$
\omega_{0} \approx \omega_{1} \ll \omega_{n}, n>1
$$

The relation $\omega_{1} \approx \omega_{0}$ is essential for the properties of macroscopic quantum tunnelling in JJs. Namely, in this case we have two channels of tunnelling, corresponding to fluxons arising near the junction's edges. This situation is considered in the next section.

\section{PROBABILITY OF PHOTON-ASSISTED TUNNELLING}

Now we calculate the probability per unit time of quantum tunnelling to another vacuum state, stimulated by external electromagnetic radiation, using the approach $\underline{9}$ proposed in one of our previous papers. In the semiclassical approximation, we can consider the quantum field $\hat{\psi}$ as a classical field $\psi(\tau, x)$ in imaginary time $t=i \tau$. The probability, $\Gamma$, then reads $\frac{12}{2}$

$$
\Gamma(\bar{E})=\omega_{p} \sqrt{\frac{30 B(\bar{E}) \mu_{0}}{\pi}} \exp [-B(\bar{E})], \quad B(\bar{E})=\frac{2 S_{E}}{\hbar},
$$

where $\mathcal{S}_{E}$ is the action (10) in imaginary time. Substituting

$$
\varphi(\tau, x)=\varphi_{0}(x)+\psi(\tau, x)
$$

into Eq. (1) and expanding the action in powers of $\psi$, we obtain

$$
\begin{aligned}
B(\bar{E})= & 2 \Lambda \int_{0}^{\tau_{0}} d \tau\left\{\int _ { - d / 2 } ^ { d / 2 } d x \left[\frac{1}{2} \psi\left(\hat{\mathcal{D}}-\frac{\partial^{2}}{\partial \tau^{2}}\right) \psi-\right.\right. \\
& \left.\left.\frac{1}{6} \sin \varphi_{0} \psi^{3}-\frac{1}{24} \cos \varphi_{0} \psi^{4}-\ldots\right]-\frac{\bar{E}}{E_{J}}\right\} .
\end{aligned}
$$

The last term in Eq. (45) originates from the matching condition for the wave function $\Phi$ of the quantum field $\hat{\psi}$ inside $\left(\Phi_{\text {in }} \propto \exp \left(-\mathcal{S}_{E} / \hbar\right)\right)$ and outside $\left(\Phi_{\text {out }} \propto\right.$ $\exp (-i \bar{E} t / \hbar))$ the barrier. The field $\psi$ in Eq. (45) satisfies the equation $\delta B(\bar{E}) / \delta \psi=0$, that is

$$
\frac{\partial^{2} \psi}{\partial \tau^{2}}-\hat{\mathcal{D}} \psi=-\frac{1}{2} \sin \varphi_{0} \psi^{2}-\frac{1}{6} \cos \varphi_{0} \psi^{3}-\ldots,
$$

with the following initial and boundary conditions

$$
\left.\frac{\partial \psi}{\partial \tau}\right|_{\tau=0, \tau_{0}}=0,\left.\quad \frac{\partial \psi}{\partial x}\right|_{x= \pm d / 2}=0
$$

We seek a solution of the equation (46) in the form

$$
\psi(\tau, x)=\sum_{n} c_{n}(\tau) \psi_{n}(x)
$$

Multiplying Eq. (46) by $\psi_{n}$ and performing space integration and using Eq. (14), we obtain the system of equations for $c_{n}(\tau)$

$$
\ddot{c}_{n}-\mu_{n} c_{n}=-\frac{1}{2} \sum_{m k} U_{n m k}^{(3)} c_{m} c_{k}-\frac{1}{6} \sum_{m k l} U_{n m k l}^{(4)} c_{m} c_{k} c_{l}-\ldots
$$

with initial conditions

$$
\dot{c}_{n}(0)=\dot{c}_{n}\left(\tau_{0}\right)=0 .
$$

Here, the dot means "imaginary time derivative", and

$$
U_{n \ldots k}^{(i)}=-\int_{-d / 2}^{d / 2} d x \frac{\partial^{i}\left(\cos \varphi_{0}\right)}{\partial \varphi_{0}^{i}} \psi_{n} \ldots \psi_{k} .
$$

The tunnelling exponent $B(\bar{E})$, Eq. (45), can be expressed as

$$
\begin{aligned}
B(\bar{E})= & \Lambda \int_{0}^{\tau_{0}} d \tau\left[\frac{1}{6} \sum_{n m k} U_{n m k}^{(3)} c_{n} c_{m} c_{k}-\frac{2 \bar{E}}{E_{J}}+\right. \\
& \left.\frac{1}{12} \sum_{n m k l} U_{n m k l}^{(4)} c_{n} c_{m} c_{k} c_{l}+\ldots\right]
\end{aligned}
$$

When the current $I$ is close to the critical value $I_{c}(d)$, we have $\mu_{0} \ll 1$ and $c_{n} \ll 1$. So, we can neglect all terms in the right-hand-side of Eq. (48), except the first one. Our analysis shows that when $d \gtrsim 4, \mu_{1} \approx \mu_{0}$, and we have the following relation for the eigenvalues of the operator $\hat{\mathcal{D}}$

$$
\mu_{0} \approx \mu_{1} \ll \mu_{n}, n>1,
$$




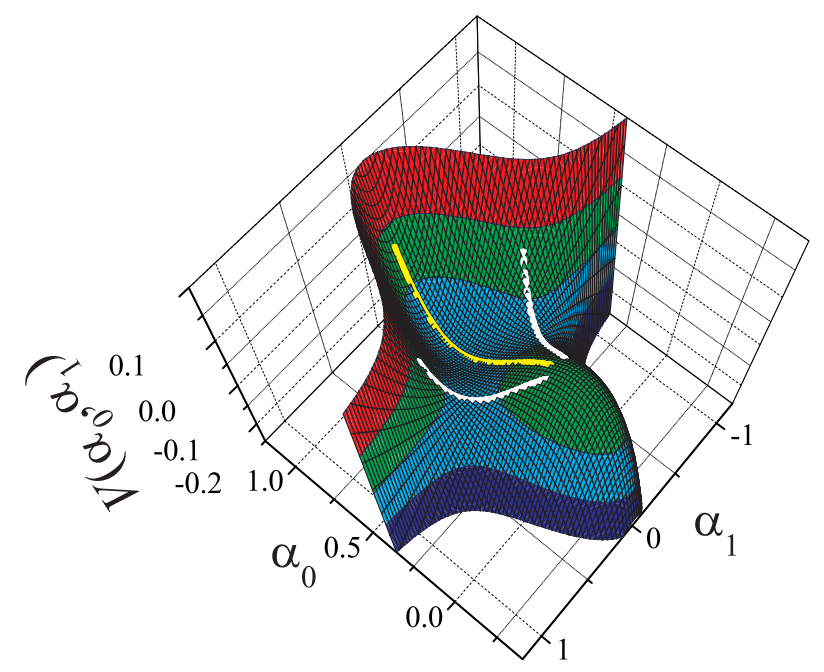

FIG. 3: (Color online) The potential $V\left(\alpha_{0}, \alpha_{1}\right)$ calculated when $d=6, I / I_{c}(d)=0.98\left(\lambda / u_{01}=1.18\right)$. A particle tunnels from its initial position near $\alpha_{i}=0$. The $\alpha_{i}$ are collective coordinates for the tunnelling fluxon. The curves correspond to three possible imaginary-time trajectories of the particle: $\alpha_{i}^{(0)}(\eta)$ and $\alpha_{i}^{( \pm)}(\eta)$ (see text below). Note, that the real-time potential equals to $-V\left(\alpha_{0}, \alpha_{1}\right)$.

In this case, $c_{0}, c_{1} \ll c_{n}(n>1)$, and we can consider only the first two equations of the system (48), taking $c_{n}=0$ for all $n>1$ (for details, see Ref. 9 ).

We now introduce new variables

$$
\alpha_{i}(\eta)=\frac{\sqrt{u_{0} u_{i}} c_{i}(\tau)}{3 \mu_{0}}, \quad i=0,1
$$

where

$$
\eta=\sqrt{\mu_{0}} \tau, u_{i}=U_{0 i i}^{(3)} .
$$

The system of equations (48) takes the form

$$
\left\{\begin{array}{c}
\frac{d^{2} \alpha_{0}}{d \eta^{2}}-\alpha_{0}=-\frac{3}{2}\left(\alpha_{0}^{2}+\alpha_{1}^{2}\right) \\
\frac{d^{2} \alpha_{1}}{d \eta^{2}}-\lambda \alpha_{1}=-3 u_{01} \alpha_{0} \alpha_{1}
\end{array}\right.
$$

where

$$
\lambda=\frac{\mu_{1}}{\mu_{0}}, u_{01}=\frac{u_{1}}{u_{0}} .
$$

The system (54) has the first integral

$$
\frac{9 \mu_{0}^{3}}{u_{0}^{2}}\left[\left(\frac{d \alpha_{0}}{d \eta}\right)^{2}+\frac{1}{u_{01}}\left(\frac{d \alpha_{1}}{d \eta}\right)^{2}+V\left(\alpha_{0}, \alpha_{1}\right)\right]=-\frac{\bar{E}}{E_{J}},
$$

where we introduce a potential

$$
V\left(\alpha_{0}, \alpha_{1}\right)=\alpha_{0}^{3}+3 \alpha_{0} \alpha_{1}^{2}-\alpha_{0}^{2}-\frac{\lambda}{u_{01}} \alpha_{1}^{2} .
$$
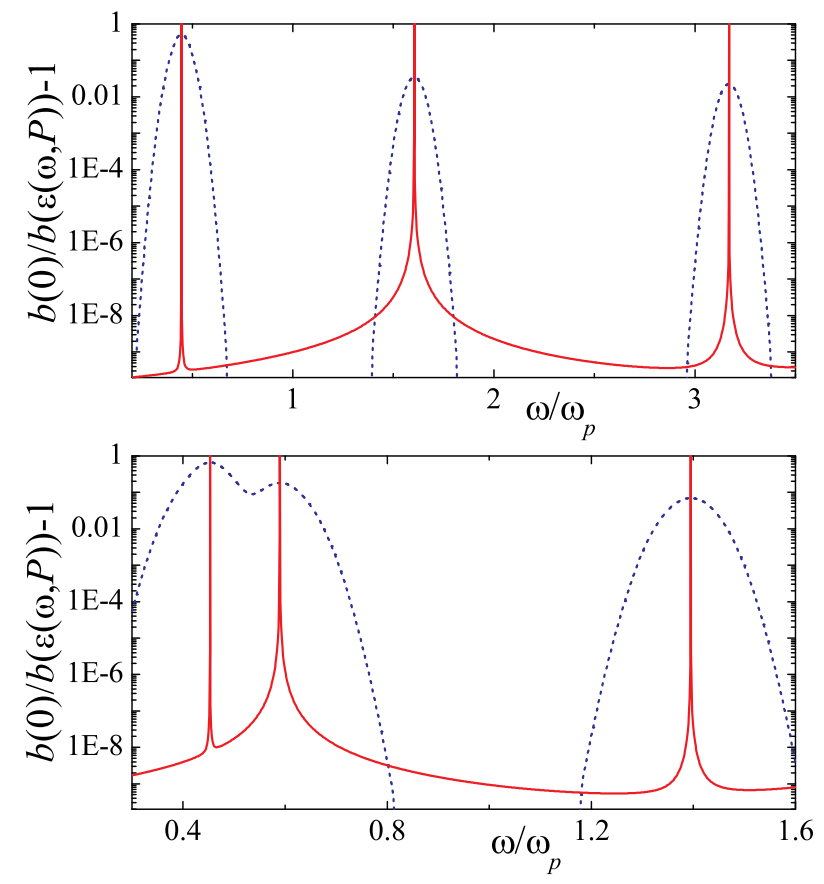

FIG. 4: (Color online) The frequency dependence of $b(0) / b(\bar{\varepsilon}(\omega, P))-1$, calculated for $d=2$ (upper graph) and $d=5$ (lower graph). Other parameters are $I / I_{c}(d)=0.98$, $L=2 \lambda_{J}$, and $v=1 / 30$ for both cases. Solid curves correspond to monochromatic incoming radiation, while the dashed curves describe the response on wide-band $\mathrm{THz}$ radiation with $\bar{\gamma}=5 \cdot 10^{-2}$ (in units of $\omega_{p}$ ). The radiation power $P$ is the same for all curves and is chosen such that $\max (\bar{\varepsilon}(\omega, P))=0.3 \varepsilon_{0}=2 / 45$ for wide-band radiation.

Taking into account the initial conditions (49), we have, at the turning points:

$$
\left.V\left(\alpha_{0}\left(\sqrt{\mu_{0}} \tau\right), \alpha_{1}\left(\sqrt{\mu_{0}} \tau\right)\right)\right|_{\tau=0, \tau_{0}}=-\bar{\varepsilon},
$$

where

$$
\bar{\varepsilon}=\frac{u_{0}^{2} \bar{E}}{9 \mu_{0}^{3} E_{J}}, \quad 0<\bar{\varepsilon}<\varepsilon_{0}=\frac{4}{27} .
$$

The equation (58) defines the value of $\tau_{0}$ as a function of system energy $\bar{E}$.

Thus, we reduce the problem of quantum tunnelling of the field $\varphi$ to the problem of tunnelling a quantum particle in two dimensions, where the $\alpha_{i}$ 's play the role of the particle generalized "coordinates". The potential $V\left(\alpha_{0}, \alpha_{1}\right)$ is shown in Fig. 3 .

When $d>d_{c}(I, \bar{\varepsilon}) \approx 4$, there are three solutions of the system of Eqs. (54) with the conditions (58), $\alpha_{i}^{(0)}(\eta)$ and $\alpha_{i}^{( \pm)}(\eta)$, which are characterized by the following relations

$$
\alpha_{1}^{(0)}(\eta)=0, \quad \alpha_{1}^{(-)}(\eta)=-\alpha_{1}^{(+)}(\eta) .
$$

The trajectories $\alpha_{i}^{(0)}(\eta)$ and $\alpha_{i}^{( \pm)}(\eta)$ are shown in Fig. 3. The solution $\alpha_{i}^{(+)}(\eta)\left(\alpha_{i}^{(-)}(\eta)\right)$ corresponds to the forma- 
tion of vortex (antivortex) nucleus at left (right) junction's edge, while the solution $\alpha_{i}^{(0)}(\eta)$ describes the tunnelling of $\varphi$ as a whole ${ }^{9}$. The minimum of $B(\bar{E})$ corresponds to the solutions $\alpha_{i}^{( \pm)}(\eta)$. The tunnelling exponent then reads

$$
B(\bar{E})=\frac{24 \Lambda \mu_{0}^{5 / 2}}{5 u_{0}^{2}} b(\bar{\varepsilon}),
$$

where

$$
b(\bar{\varepsilon})=\frac{15}{16} \int_{0}^{\eta_{0}} d \eta\left[\alpha_{0}^{3}+3 \alpha_{0} \alpha_{1}^{2}-2 \bar{\varepsilon}\right], \quad \eta_{0}=\sqrt{\mu_{0}} \tau_{0} .
$$

Note, that we should multiply the probability $\Gamma(\bar{E})$, Eq. (44), by a factor of 2 , since we have two channels for tunnelling.

When $d<d_{c}(I, \bar{\varepsilon})$, all three solutions coincide, $\alpha_{1}^{(0)}(\eta)=\alpha_{1}^{(-)}(\eta)=\alpha_{1}^{(+)}(\eta)=0$, and the second equation of the system (54) becomes trivial, while the first one can be easily integrated. As a result, we obtain

$$
b(\bar{\varepsilon})=\frac{15}{16} \int_{\alpha_{1}(\bar{\varepsilon})}^{\alpha_{2}(\bar{\varepsilon})} d \alpha \frac{\alpha^{3}-2 \bar{\varepsilon}}{\sqrt{\alpha^{2}(1-\alpha)-\bar{\varepsilon}}}, \quad b(0)=1,
$$

where $\alpha_{1,2}(\bar{\varepsilon})$ are the smaller and larger positive roots of the cubic equation

$$
\alpha^{2}(1-\alpha)-\bar{\varepsilon}=0 .
$$

The analysis of the tunnelling exponent $B$ on the junction's width $d$ and current $I$ was carried out in one ${ }^{9}$ of our previous papers. Now we are interested in the effect of electromagnetic radiation on $B(\bar{E})$. Using formulas (40), (59), (61), and (62), we calculate the dependence of $b(0) / b(\bar{\varepsilon}(\bar{\omega}, P))-1$ as a function of radiation's central frequency $\bar{\omega}$, for short $d<d_{c}$, and long $d>d_{c}$ junctions. The results of the calculations, both for wideband $\left(\bar{\gamma} \gg \gamma_{n}\right)$ and monochromatic $\left(\bar{\gamma} \ll \gamma_{n}\right)$ radiation, are shown in Fig. 4. It is clear that we have several resonances at frequencies $\bar{\omega}=\omega_{n}=\sqrt{\mu_{n}}$ (in units of $\omega_{p}$ ). When $\bar{\gamma}=0$, the resonance peaks are very narrow, and one can switch the JJ to the resistive state $(B=0)$ at small radiation power, if $\bar{\omega}=\omega_{n}$. Note, that the condition $\bar{\omega}=\omega_{n}$ can be achieved by changing the applied dc current $I$, since $\omega_{n}$ depend on $I$. In other words, if the frequency of the incoming radiation lies near one of the $\omega_{n}$, one can observe a resonance behavior of the tunnelling exponent $B$ as a function of the dc current.
For relatively short junctions, $d \lesssim 1$, we have several well separated peaks even for wide-band $\mathrm{THz}$ radiation, as it can be seen from Fig. 45 while for $d \gtrsim d_{c}$, we have $\omega_{0} \approx \omega_{1} \ll \omega_{n}$, and the first two peaks can merge into a single peak. Note, that the inequality $\omega_{0} \approx \omega_{1} \ll \omega_{n}$ is valid for not too high junction's width $d \lesssim 20$. In the opposite case we should consider the large number of equations in the system (48) to calculate the tunnelling exponent.

\section{CONCLUSION}

In conclusion, we proposed a quantum field theory for Josephson plasma waves interacting with external electromagnetic waves. We also calculate the macroscopic quantum tunnelling of a fluxon, stimulated by $\mathrm{THz}$ light, in a long Josephson junction driven by a dc current. The probability of absorption and emission of light depends on the current and the length of the Josephson junction. The MQT escape rate shows several resonance maxima as a function of the frequency, corresponding to eigenfrequencies of Josephson plasma wave quanta. This could be potentially useful for a variety of superconducting quantum $\mathrm{THz}$ devices. Classical $\mathrm{THz}$ devices are discussed in Ref. 14 .

\section{Acknowledgements}

We acknowledge partial support from JSPS-RFBR 0602-91200.

FN and SS acknowledge partial support from Core-toCore (CTC) program supported by the Japan Society for Promotion of Science (JSPS).

FN gratefully acknowledges partial support from the National Security Agency (NSA), Laboratory Physical Science (LPS), Army Research Office (ARO), National Science Foundation (NSF) grant No. EIA-0130383.

SS acknowledges support from the Ministry of Science, Culture and Sports of Japan via the Grant-in Aid for Young Scientists No 18740224, the UK EPSRC via No. EP/D072581/1, EP/F005482/1, and ESF networkprogramme "Arrays of Quantum Dots and Josephson Junctions".

AO acknowledges partial support from the Russian Foundation for Basic Research (RFBR) (grant No. 0602-16691), and Russian Science Support Foundation. AO also acknowledges prof. A. L. Rakhmanov for fruitful discussions.
1 S. Takagi, Macroscopic Quantum Tunnelling (Cambridge University Press, Cambridge, 2002).

2 J. Q. You and F. Nori, Phys. Today 58, No 11, 42 (2005).

${ }^{3}$ K. Inomata, S. Sato, K. Nakajima, A. Tanaka, Y. Takano,
H. B. Wang, M. Nagao, H. Hatano, and S. Kawabata, Phys. Rev. Lett. 95, 107005 (2005).

4 T. Bauch, F. Lombardi, F. Tafuri, A. Barone, G. Rotoli, P. Delsing, and T. Claeson, Phys. Rev. Lett. 94, 087003 
(2005).

5 T. Bauch, T. Lindstrom, F. Tafuri, G. Rotoli, P. Delsing, T. Claeson, and F. Lombardi, Science 311, 57 (2006).

6 X. Y. Jin, J. Lisenfeld, Y. Koval, A. Lukashenko, A. V. Ustinov, and P. Müller, Phys. Rev. Lett. 96, 177003 (2006).

7 S. Savel'ev, A. L. Rakhmanov, and F. Nori, Phys. Rev. Lett. 98, 077002 (2007); Erratum: 98, 269901 (2007).

8 Animations illustrating macroscopic quantum tunnelling in long junctions can be found online in dml.riken.jp/MQT

9 A. O. Sboychakov, S. Savel'ev, A. L. Rakhmanov, and F. Nori, Europhys. Lett. 80, 17009 (2007).

10 S. Savel'ev, A. O. Sboychakov, A. L. Rakhmanov, and F. Nori, Phys. Rev. B 77, 014509 (2008).

11 A. O. Caldeira and A. J. Leggett, Phys. Rev. Lett. 46, 211 (1981).

12 S. Coleman, Phys. Rev. D 15, 2929 (1977).

13 V.B. Berestetskii, E. M. Lifshitz, and L. P. Pitaevskii, Quantum electrodynamics (Pergamon Press, Oxford, 1982).
14 S. Savel'ev et al., Nature Phys. 2, 521 (2006); Phys. Rev. Lett. 95, 187002 (2005); Physica C 445-448, 183 (2006); Phys. Rev. B 75, 184503 (2007); Phys. Rev. Lett. 94, 157004 (2005); Phys. Rev. B 74, 184512 (2006); Phys. Rev. B 72, 144515 (2005); Phys. Rev. E 72, 056136 (2005); Physica C 445-448, 180 (2006); Physica C 437-438, 281 (2006); Physica C 445-448, 175 (2006); V.A. Yampol'skii et al., Phys. Rev. B 75, 014527 (2007); Phys. Rev. B 76, 224504 (2007).

15 M. Machida, T. Koyama, Physica C 463, 84 (2007).

16 The action (1) has also a surface term, proportional to $\oint_{\Sigma} d \zeta \varphi_{0}\left[\operatorname{rot} \mathbf{A} \times \mathbf{e}_{z}\right]_{n}$. Since we consider an ac magnetic field, the time-average of this term is zero, and we neglect it.

17 We do not consider the process of spontaneous absorption since the intensity of re-emitted photons by the junction is assumed to be negligible in comparison to the intensity of incoming radiation. 